\title{
Role of beliefs and emotions in numerical problem solving in university physics education
}

\author{
Madelen Bodin* \\ Department of Physics, Umeå University, Umeå, Sweden \\ Mikael Winberg ${ }^{\dagger}$ \\ Department of Science and Mathematics Education, Umeå University, Umeå, Sweden
}

(Received 31 May 2011; published 29 February 2012)

\begin{abstract}
Numerical problem solving in classical mechanics in university physics education offers a learning situation where students have many possibilities of control and creativity. In this study, expertlike beliefs about physics and learning physics together with prior knowledge were the most important predictors of the quality of performance of a task with many degrees of freedom. Feelings corresponding to control and concentration, i.e., emotions that are expected to trigger students' intrinsic motivation, were also important in predicting performance. Unexpectedly, intrinsic motivation, as indicated by enjoyment and interest, together with students' personal interest and utility value beliefs did not predict performance. This indicates that although a certain degree of enjoyment is probably necessary, motivated behavior is rather regulated by integration and identification of expertlike beliefs about learning and are more strongly associated with concentration and control during learning and, ultimately, with high performance. The results suggest that the development of students' epistemological beliefs is important for students' ability to learn from realistic problem-solving situations with many degrees of freedom in physics education.
\end{abstract}

DOI: 10.1103/PhysRevSTPER.8.010108

PACS numbers: 01.40.Fk, 01.40.Ha, 01.50.H-

\section{INTRODUCTION}

In recent years there has been an increasing interest in using numerical methods including interactive simulations and visualizations in university physics education [1-3]. Increasing computer capacity together with a number of modeling and problem-solving environments offer new possibilities for physics students to model complex physics systems as well as to numerically solve physics problems. Depending on the modeling environment, students are given different opportunities to numerically solve physics problems, create visual simulations, practice mathematical modeling, and investigate the process of a physics phenomena, rather than just focusing on an answer [3-7].

There is not, however, much research on how the students deal with this type of problem solving and how the computer as a cognitive and computational tool affects students' learning. Many, but not all, simulation and modeling environments represent learning situations that offer many degrees of freedom and open up avenues for students to take control of their

\footnotetext{
*Author to whom correspondence should be addressed. madelen.bodin@physics.umu.se

†mikael.winberg@matnv.umu.se

Published by the American Physical Society under the terms of the Creative Commons Attribution 3.0 License. Further distribution of this work must maintain attribution to the author(s) and the published article's title, journal citation, and DOI.
}

own learning, an approach which is expected to be favorable for motivation to learn [8]. Computer environments that require independence and creativity might, however, have different effects on different students. Research shows that the student's personal features, such as prior knowledge and beliefs, that are brought into a specific learning situation affect both learning process and outcomes of a learning situation $[9,10]$ and influence the degree to which the student becomes externally or internally motivated [8]. Emotions have been proposed to have a central role in the evaluation and regulation of behavior as well as functioning as goals or desired end states of behavior [11,12]. Students' beliefs about utility of science and studying science have in several studies been suggested to play an important role [13] which, together with students' expectancies of success, has been claimed to be one of the primary predictors of motivation [14]. In this study, we have focused on investigating the relation between university students' personal characteristics (prior knowledge and beliefs) and perceived emotions and their effect on learning outcome in terms of performance in a learning situation involving numerical physics problem solving and creation of physics simulations in the field of classical mechanics. In order to describe the background variables, the conceptual framework in this work is based on the following research fields: modeling and simulations in physics education, the role of beliefs on physics learning, and emotions and motivational aspects on learning. 


\section{CONCEPTUAL FRAMEWORK}

\section{A. Modeling and simulations in physics education}

Modeling forms the basis for doing physics, i.e., describing physics phenomena and solving physics problem. Hestenes [15] suggests that students must train to make and use models for specific physics systems and processes in order to learn the structure of physics. Computer environments for modeling and simulations have shown to be powerful tools in physics education for developing conceptual understanding as well as for modeling and problem-solving skills [3-7]. However, modeling environments can represent different levels of autonomous problem-solving approaches and require more or less knowledge and skills in modeling, programming, and mathematics, as well as conceptual physics. A task that requires advanced mathematical modeling and graphics programming, e.g., a programming task in $\mathrm{C}++$, is expected to impose other demands on the student, and give other learning outcomes, than a task designed for a sandbox environment where the simulation can be built using a graphics interface, e.g., ALGODOO [16]. In between pure programming and sandbox tools, we have environments that to different degrees provide the scope for intellectual autonomy, require active participation from the student, and give possibilities to actually model physics systems and create interactive simulations. For example, tools like Easy JAVA Simulation environment [4] and VPYTHON [7] allow the student to model physics problems by setting up the physics and mathematics needed to describe the simulation and create visual interactive simulations. Problem-solving environments, such as MATLAB and MAPLE, provide some support concerning functions and graphics, but also require programming skills in order to numerically solve a physics problem and visualize the result in a simulation [17].

The level of autonomy in a learning situation has been suggested as an important situational variable, depending on the degree of internalization of instructional goals, and hence the level and type of motivation to engage in the task and the quality of learning outcomes [8]. Autonomy is a multidimensional construct and can take different forms depending on, e.g., stage of learning and context, but a general description is the learners' possibility to take charge or control of their own learning. The level of autonomy of a task can be related to the task's degree of freedom, i.e., how many choices a student has to take control of the situation, e.g., when, where, and how the task can be done, but is also determined by the student's perceived autonomy, indicated by the student's perceived control and competence. How much control the student is capable of handling is also dependent on the student's knowledge and skills. Benson [18] suggests that autonomous behavior is essentially self-initiated rather than generated in response to a task. Teaching strategies are also critical and have to be taken into consideration when providing the student with help and support through tasks with many degrees of freedom in order to optimize learning [19]. The level of autonomy characteristics of a task, in terms of degrees of freedom, might thus affect students' performance differently depending on students' characteristics, in terms of knowledge, beliefs, and motivation. There is therefore a need to examine the relationships between these variables more thoroughly.

\section{B. Beliefs about knowledge and learning}

An interest in students' beliefs about the nature of knowledge, where it resides, and how it is constructed and evaluated is common in contemporary research on epistemological beliefs. There are a number of views on how epistemological beliefs should be defined and measured, and therefore their relation to learning is still ambiguous [20,21]. Perry's [22] work on the intellectual and ethical development of college students is considered by many as the starting point for research on epistemological beliefs. Perry proposed that student's beliefs about several aspects of learning could be collapsed into one single continuous component describing a development from dualistic, viewing knowledge as absolute and transferable, to a more relativistic view, where their own responsibility in the process of learning and the contextdependent nature of knowledge is acknowledged, corresponding to a more sophisticated epistemology. Schommer [23] assumed that the construct of epistemological beliefs comprised five independent dimensions. Three of these belief dimensions can be seen as adaptations of Perry's work: simplicity of knowledge (e.g., knowledge consists of discrete facts, right or wrong), certainty of knowledge (i.e., knowledge is certain versus tentative and context dependent), and source of knowledge (from authorities versus constructed by the student). The remaining two dimensions were pertinent to student's beliefs about the speed of learning (quick or not at all) and the nature of the ability to learn. In physics education epistemological beliefs are often considered on a noviceexpert continuum where the novice has dualistic beliefs of seeing physics as isolated pieces of information with no connection which eventually will develop towards expert, relativistic beliefs where physics consists of general concepts and principles [24]. Winberg and Berg [25] found that this dualistic-relativistic, or novice-expert, beliefs continuum was useful in predicting complexity as well as correctness of university student's chemical reasoning. Hammer [24], using a framework consisting of three dimensions, structure of physics, content of physics knowledge, and learning physics, found that students' beliefs affected their learning in the course and were consistent across physics content. If, for example, students believed that physics knowledge consists of isolated pieces, this was reflected in how students related results to specific facts rather than general principles. 
Elby and Hammer [26] challenged the consensus that sophisticated epistemology constitute a tentative, independent, and coherent view forming relativistic beliefs about knowledge and learning, as, for example, described by Hofer and Pintrich [9] and applied in Schommer's instrument measuring epistemological beliefs [23]. Elby and Hammer argue that when assuming a sophisticated epistemology it is necessary to also consider the context in which the epistemologies are measured, e.g., within which discipline, the particular knowledge, and the intended use of the knowledge.

Surprisingly little attention has been devoted to the interaction between students' epistemological beliefs and situational factors and how this interaction affects motivation. One exception is a study by Windschitl and Andre [10] who found that a constructivist simulation environment had positive effects on the performance on a subsequent test on conceptual understanding for students with relativistic beliefs, while students with dualistic beliefs were negatively affected. When exposed to a simulation context that provided less scope for cognitive autonomy, students with dualistic beliefs instead outperformed students with relativistic beliefs on the subsequent knowledge test. The authors suggested that the mismatch between the characteristics of the task and the students' relativistic beliefs about learning affected their motivation and, eventually, performance. The need for matching the situational characteristics with students' beliefs about learning has also been pointed out by Redish [27], who argued that the degree of autonomy must be matched to students' epistemological beliefs for the situation to produce positive effects with respect to cognition and motivation. In a similar fashion, Finster [28,29] argued that a mismatch between the offered context for learning, e.g., complexity and degrees of freedom of the intellectual task, and the learner's epistemological beliefs will result in students becoming either overwhelmed or not challenged enough, depending on which way the mismatch goes, but in both cases having negative influence on students' motivation to learn as well as cognition.

While epistemological beliefs are ideas about the character of knowledge and the process of learning, beliefs may also express what value the outcome of the learning activity has for a student. This value is evaluated in relation to whether the knowledge or learning activity will lead to the achievement of a goal, e.g., passing an exam, improve future job opportunities, enhance self-image, or generate positive emotional experiences $[8,11]$. In general, studies indicate that value beliefs are primarily linked to choice behavior, i.e., motivation to engage in a task [14]. However, there are indications that perceived usefulness of physics knowledge for future career goals influences students' readiness to realize and resolve cognitive conflict [30] as well as their goal orientation and learning strategies [31]. Since engagement in learning, as well as choices of learning strategies, would involve thoughts about the nature of knowledge and how learning is achieved, value beliefs could be related to students' epistemological beliefs as well as to motivation to engage in the activity of learning physics. In a work by Adams et al. [32] an instrument was developed in order to investigate student beliefs about physics and learning physics and to distinguish between experts and novices in order to predict performance. The instrument covers epistemological as well as value beliefs and is context dependent. The statements assume a more practical view rather than the dualistic-relativistic view adapted in previous instruments [23] covering alternative dimensions of beliefs as suggested by Elby and Hammer [26]. The statements are empirically categorized, based on student responses, resulting in test items measuring epistemological beliefs, reflecting cognitive aspects, as well as value beliefs, reflecting attitudes towards physics. In order to develop the knowledge base about beliefs there is still a need to further examine the relationship between epistemological and value beliefs as well as their correlation with cognition and motivation.

\section{Motivation and emotions}

Motivation is commonly viewed as the process whereby a goal-directed activity is instigated and sustained [33]. Common ways to assess an individuals' motivation to engage in a specific activity is to investigate the individual's behavior, e.g., choice and persistence. However, motivational frameworks frequently describe emotions as emerging from appraisals of self-competence in relation to the task [34], locus of causality as well as perceived importance of successful performance on the task for goal attainment [12], rate of progress in goal attainment, and to what extent the situation provides scope for autonomy [8,11]. Positive activating state emotions, such as hope and enjoyment, are assumed to lead to more effort, deeper cognitive engagement, self-regulation, and less irrelevant thinking in academic settings [35]. In theories pertinent to the intrinsic-extrinsic motivation continuum, such as selfdetermination theory [8], intrinsic motivation is commonly described as a will to engage in an activity because it elicits positive emotions, e.g., satisfaction and enjoyment, as a contrast to extrinsic motivation, which is considered to emerge from appraisals of the instrumental value of the activity [36]. Ryan and Deci [8] proposed a more elaborate model where extrinsic motivation could vary in terms of the degree of internalization and integration of extrinsic goals. In this model, an increased internalization of goals is accompanied by a sense that behavior is self-determined, i.e., related to autonomy, which in turn is proposed to lead to increased persistence, more positive emotional experiences during the activity, and higher quality of engagement. However, Ryan and Deci [8] argued that for autonomy to lead to more intrinsic forms of motivation, it has to be paralleled by a sense of competence, or 
self-efficacy, since goals are more likely to be internalized if the individual thinks he or she has the skills necessary to attain them. Csíkszentmihályi [37] proposed that appraisals of one's own skills in relation to the challenges in the situation cause emotions that in turn regulate behavior. Flow, an intense feeling of active well-being, corresponds to the goal and is elicited when skills and challenge are above threshold level and in balance. If flow is within reach, emotions like control or excitement function as activators that encourage the individual to increase their skills or the level of challenge to achieve balance. If the discrepancy between skills and challenge are too large, emotions like boredom, anxiety, uneasiness, or relaxation interrupt behavior, which allows the individual to attend to a new goal or activity.

According to Carver and Sheier [11] and their controlprocess model, epistemological beliefs could also function as goals that emanate from a more overriding image of what kind of student the individual wants to be. Consequently, epistemological beliefs would not only relate to how students approach the task, from a cognitive aspect, but also the emotional experiences that result from students' assessment of how the results of their learning behavior, i.e., the implementation of their beliefs about learning, contribute to, or align with, their desired selfimage. Thus, as previously pointed out, the match between students' epistemological beliefs, on one hand, and the intellectual demands of the situation, on the other, is likely to have correlations with student emotional experiences and thus motivation to engage in a task.

\section{Research questions}

In the conceptual framework above we have discussed the different constructs that have, in previous research, shown to be important in a learning situation. Personal factors, e.g., prior knowledge and beliefs, as well as situational factors, e.g., task characteristics and perceived emotions, are two dimensions that affect how students learn, and in order to further understand how these constructs affect learning, we have investigated the interrelations between the constructs and the performance of a physics task which provides autonomy characteristics, such as extensive mathematical modeling as well as possibilities to take charge of when and where to solve the task.

We have thus investigated students' characteristics, in terms of prior knowledge and beliefs, and emotional experiences in the context of a learning situation with autonomy characteristics where university students numerically solve and simulate a physics problem. The research question is, What are the relationships between students' prior knowledge, epistemological and value beliefs, and emotional experiences (control, concentration, and pleasure) and how do they correlate with the quality of performance in a learning situation with many degrees of freedom?

\section{METHOD}

\section{A. Participants and context}

The context for this study was limited to a one-week assignment which was part of a regular course for physics students. Students participating in this study were second year university physics students taking a 7-week full-time course in mathematics of physical models. The course was compulsory for the 4.5-year study program in Master of Science in engineering physics. The students were initially informed about the study by Email from us and participation was voluntary. The assignment chosen for the study was a regular assignment in the course, a scheduled oneweek project which actually lasted for several weeks due to winter break. We were only researchers in this study and did not have any teaching roles. The course had 50 registered participants, of which 33 men and 7 women chose to contribute to the data set collected in the study. Those who did not contribute were either not present at the time for data collection or chose to not contribute to the data set. The statistical method chosen for analysis (principal component analysis) does, however, tolerate missing values at this level and is described in more detail below. The final model was based on the 30 students that turned in the lab report. The mean age of the participants was 23 years.

The assignment consisted of mathematical modeling of an elastic object sliding over a rough surface using the particle-spring model. The particle-spring model is a fundamental model system in science and can be used to model many science phenomena with vibrations and oscillations. The purpose of this simulation task was to train students to build mathematical models of a physics situation and to create a visual simulation of that situation. The project consisted of four successive subtasks. The first subtask introduced the student to the model used in the simulation: two mass particles connected by a spring. In the following subtasks more particles and springs were added in order to model a larger system. At completion of the four subtasks, the student would have built a simulation of a macroscopic elastic object sliding over a rough surface in two dimensions. The problem-solving environment used for the project was MATLAB. Prior to this course the students had several occasions to learn about and work with MATLAB in different courses in their study program. The physics that was needed in order to understand and perform the assignment was classical mechanics, a course that all students had completed. An introductory lecture was held before the MATLAB project started which was composed of a short summary of the physics of the simulation and a brief introduction to the simulation methods that would be suitable for this task. The first milestone occurred after one week when the students could earn bonus marks for the exam by delivering a preliminary, but functioning, code for the simulation. The deadline for the full report was due four weeks after the project started, with Christmas holidays between the preliminary report 
and the final deadline. To pass the assignment, students were required to submit a report including answers to a set of questions in their instructions, a description of how the simulation was tested, and the MATLAB code for the simulation. The task allowed the students to create their own solutions and work at their own pace. The students could choose whether to use the university computer lab and support from teacher assistants or do the task at some other place. The students thus had several possibilities to take control of their own learning and the learning situation was considered as having many degrees of freedom.

\section{B. Procedure}

Data were collected for four different variables, i.e., prior knowledge, beliefs, emotions, and performance. Students' responses to questionnaires and lab reports were the main sources of data in this study. Students' beliefs and prior knowledge were measured at the time of the project start. Emotions were measured during the first week of the project at two occasions. Lab reports were collected and analyzed after the final deadline, which was four weeks after the project start. Within these four weeks there were two weeks of holidays. Each data-collecting instrument is described in more detail below. Responses from all tests and questionnaires were analyzed by multivariate analysis methods to find the main sources of variation between students and to characterize them from the aspects reported in the preceding section.

\section{Data collection instruments}

Prior knowledge.-The conceptual test was given in order to map the student's conceptual prior knowledge in classical mechanics. The test consisted of 12 multiplechoice questions, originating from the Force Concept Inventory test [38] and the Force and Motion Conceptual Evaluation test [39]. The test items were chosen in order to represent the basic conceptual knowledge in classical mechanics needed in order to understand and solve the task. Thirty-two students responded to this test.

Beliefs.-For measurements of beliefs we used the Colorado Learning Attitudes about Science Survey (CLASS), developed from a number of existing surveys, probing beliefs about physics, and learning physics by Adams et al. [32]. The CLASS survey is designed in order to distinguish between expert and novice beliefs about physics. The original survey consists of 42 statements that claim either expertlike or novicelike beliefs. The corresponding responses are collected on a five-degree Likert scale, ranging from strongly agree to strongly disagree. From these statements, Adams et al. [32] used a combination of multivariate analysis and face validity judgements by expert physicists to identify eight categories labeled real world connections, personal interest, sensemaking effort, conceptual connections, applied conceptual understanding, problem solving general, problem solving confidence, and problem solving sophistication. Before using the survey in this study we translated all statements into Swedish. Three questions were omitted since Adams et al. [32] have questioned their validity, resulting in a 39 -item questionnaire. There were 32 students who responded to this questionnaire. The beliefs questionnaire was administered once at the start of the project assignment. The purpose was not to monitor changes in students' beliefs since these were expected to be fairly stable during the short period of time this assignment lasted.

Emotions.-The items used in this study are based on a questionnaire constructed by Ghani and Deshpande [40] measuring flow experiences in human-computer interaction. Thirty-five students contributed to the emotions data. The items are chosen to assess the flow components control (control, calm, competent, ignorant, frustrated, stressed), concentration (concentration, focus, absorbed), and pleasure (interested, amused, motivated, elated). During their work on the simulation task students were, at two separate occasions about two days apart, asked to rate to what degree they experienced each of these 13 emotions on a five-degree Likert scale, ranging from very much to not at all. Questionnaires were administered using a web application.

Lab report.-Each student turned in a written lab report after completing the assignment. The students were instructed to provide a well-functioning code for the simulations, answers to a number of questions concerning conceptual physics and numerical aspects of the problemsolving process, and provide graphs with explanations representing energy calculations from the simulation. The assignment consisted of four successive subtasks, of which the last three were treated in the lab report as answers to questions about conceptual physics as well as about the numerical solutions. The subtasks corresponded to increasing complexity of the assignment, from modeling of a small particle-spring system towards large particle-spring systems including damping. The questions associated with these steps differ in terms of required answers. In subtask 2, the answers to explicit questions were required in the lab report while in subtasks 3 and 4 the students had greater possibilities to choose scope and complexity when discussing their results. In order to get a measure of the quality of the reports, we used a SOLO-like taxonomy. The SOLOtaxonomy was developed by Biggs and Collis [41] as an instrument for measuring quality of learning, based on how many arguments, i.e., concepts and ideas, that are used, and how they are used, when performing a particular task. Thus, the different stages in the SOLO taxonomy can be regarded as illustrating an increasing complexity of a student's understanding of a subject. The prestructural stage represents missing the point or avoiding the question; the unistructural stage means selecting one argument and close the response with that; in the multistructural stage the student may include several concepts in their response but 
TABLE I. Example of SOLO analysis of lab score.

\begin{tabular}{lcc}
\hline \hline SOLO & Level & Question: Do you get the same score and value if you solve the equation analytically? \\
\hline 1 & Prestructural & Answer limited to, e.g., analytical value corresponds well with the numerical. \\
2 & Unistructural & Answer with either value or plot. \\
3 & Multistructural & Answer with value or plot. Comparison between numerical and analytical value. \\
4 & Relational & Answer with value or plot. Comparison between numerical and analytical value. \\
& Relates result to previous questions in the lab instruction. \\
5 & Extended abstract & Answer with value or plot. Comparison between numerical and analytical value. \\
& Relates result to previous questions in the lab instruction. Extending discussion to \\
& effects of approximations in the numerical solution.
\end{tabular}

makes no integration between them; the relational stage refers to when the student is able to use all relevant information and integrate it within the context of the question; and finally in the extended abstract the student can relate relevant information with abstract reasoning beyond the context of the question. An example of the scoring procedure is shown in Table I. Each answered question in the lab report was treated as a unit of analysis and graded from 1 to 5 according to the criteria of the analysis protocol. This resulted in a total of 15 variables that were weighed together according to their discriminating ability and linearly combined into one lab report score, expressing the students' performance, by the use of principal component analysis (PCA). The quality of the programming code was not analyzed. We performed analysis using an iterative strategy in order to get a reliable measure of the quality of the lab report. Discrepancies in coding were continuously discussed in order to establish a common ground regarding the assignment of grades. A total of 30 lab reports were turned in by the students and subject for coding. Twenty of the lab reports were coded by both of us and the Cronbach's alpha for interrater reliability was 0.90 .

\section{Statistical analysis}

In order to, to some extent, circumvent the statistical problems associated with the high degree of multicollinearity inherent in matrices that have more variables than observations and the noise that is usually present in questionnaire data, we have chosen to use projection methods such as PCA and its regression counterpart, partial least squares analysis (PLS), in our multivariate analyses [42]. The statistical software used for these analyses was SIMCA-P+ [43].

In this study, PCA has been used for reduction of the rather large number of initial variables (i.e., the individual test and questionnaire items) into a lower number of constructs to facilitate interpretation of the relationships between the studied variables and to increase reliability and validity of the instruments. PCA summarizes any number of original variables by linearly combining them into a lower number of components. Thus the components can be viewed as latent variables that express the common information contained in the original variables. Hence, questionnaire items that have been designed to measure a single theoretical construct can be summarized by one or several (if the construct is multidimensional) components, and the students' score on the theoretical construct, i.e., the principal component, can be calculated from his or her responses to the original items. The meaning of the component is interpreted by assessing the loading pattern of the original items on the principal component. Items that contribute much to the meaning of the component have high loadings while items whose variance is only to a small part captured by the component, and thus only partly contributing to the definition of the meaning of the component, have lower loadings. In the present paper, questionnaire items were grouped according to the constructs or categories previously defined and validated by Adams et al. [32], and separate PCA models were built in order to determine the relative scores of students on the different constructs. In a similar fashion, students' performance on the different questions in the pretest, their responses to flow emotion items, and lab scores, were subjected to separate PCAs (Table II). To compensate for differences in units of measurement and thus magnitude of variance, all variables were scaled to unit variance and centered. Hence, for each variable, e.g., questionnaire item, responses were expressed as the difference between the observed and the average response, divided by the standard deviation for the specific variable. Crossvalidation [44] was used to determine the significance of the principal components, and hence the number of components to retain. If not otherwise stated, the crossvalidation limits in SIMCA for retaining a component correspond to $p<0.05$, which means that including the component leads to a significant increase in the model's predictive ability.

Hierarchical PLS analysis was used to investigate the multivariate covariation structures between different constructs, such as beliefs and attitude categories and emotional experiences, as well as how all these together contribute to explaining the cognitive outcome of the exercise, i.e., the lab report score. Put briefly, hierarchical PLS utilizes students scores on the components in PCA models of each construct, i.e., base models, rather than their 
TABLE II. Statistics of PLS top model shown in Fig. 1 where outcome (lab score) is predicted by all other constructs, together with statistics of the PCA base models that represent each construct.

\begin{tabular}{|c|c|c|c|c|c|c|}
\hline & No. of components & No. of items & $R^{2} x^{\mathrm{a}}$ & $R^{2} y^{\mathrm{b}}$ & $Q^{2 \mathrm{c}}$ & Significant $^{\mathrm{d}}$ \\
\hline \multicolumn{7}{|l|}{ PLS top model } \\
\hline Lab score versus all other constructs & 1 & 13 & 0.43 & 0.42 & 0.33 & Yes \\
\hline \multicolumn{7}{|l|}{ Construct (PCA base model) } \\
\hline Prior knowledge & 1 & 13 & 0.37 & & 0.10 & Yes \\
\hline Beliefs: Real world connection & 1 & 4 & 0.46 & & 0.01 & No \\
\hline Beliefs: Personal interest & 1 & 6 & 0.33 & & -0.10 & No \\
\hline Beliefs: Sense-making effort & 1 & 7 & 0.42 & & 0.15 & Yes \\
\hline Beliefs: Conceptual connections & 1 & 6 & 0.34 & & -0.02 & No \\
\hline Beliefs: Applied conceptual understanding & 1 & 7 & 0.27 & & -0.10 & No \\
\hline Beliefs: Problem solving general & 1 & 8 & 0.33 & & -0.06 & Yes \\
\hline Beliefs: Problem solving confidence & 1 & 4 & 0.43 & & -0.07 & No \\
\hline Beliefs: Problem solving sophistication & 1 & 6 & 0.44 & & 0.15 & Yes \\
\hline Emotions: Control & 1 & 12 & 0.44 & & 0.26 & Yes \\
\hline Emotions: Concentration & 1 & 6 & 0.66 & & 0.39 & Yes \\
\hline Emotions: Pleasure & 1 & 8 & 0.55 & & 0.32 & Yes \\
\hline Lab score & 1 & 15 & 0.44 & & 0.33 & Yes \\
\hline
\end{tabular}

${ }^{\mathrm{a}}$ Variance in $x$.

${ }^{\mathrm{b}}$ Variance in $y$.

${ }^{\mathrm{c}}$ Predictive ability.

${ }^{\mathrm{d}}$ Significant component if $Q^{2}>$ limit, with $95 \%$ confidence interval. PLS: limit $=0.05$. PCA: The limit increases with subsequent components to account for the loss in degrees of freedom.

original responses to the questionnaire and test items. In PLS, separate PCA models are built on the dependent and independent variables, respectively, while simultaneously optimizing the correlation between the components of the two models. Hence, PLS reveals the correlation structure between the independent and dependent variables, respectively, as well as how the independent variables together can explain or predict the dependent variables. Some information is by necessity lost when students' responses to the original items are summarized by a smaller number of principal components. To check if this affected the relationships of interest, we also performed a PLS analysis on the original items (not shown) and compared it to the PLS top model (Fig. 1). This comparison gave no indication that data reduction had changed the overall picture. To further aid the interpretation of the relative importance of the different independent variables for predicting the outcome, variable importance for projection (VIP) plots were made (e.g., Fig. 2). These plots show the relative importance of the variables for predicting the lab report outcome. This is calculated by summarizing the loadings of each variable on the components in the PLS model, weighted by the proportion of variation in the outcome that is explained by the respective components.

\section{RESULTS}

Despite small sample size, the results support the construct, predictive, and concurrent validity of instruments used in the study. Patterns of covariation between items are intelligible from a theoretical perspective. The constructs used for investigation correspond to the data collection instruments: prior knowledge, beliefs, emotions, and lab score. The base models that were made to represent these constructs are described in more detail below. The relationships between constructs and how they can be used to predict performance is investigated in the PLS top model in Fig. 1.

\section{A. Prediction of performance by prior knowledge, beliefs, and emotions: Top model}

Figure 1 displays the PLS top model including base models of all the measured constructs. It describes the internal relationships between the different belief categories, prior physics knowledge, and emotions, and their correlation with the outcome variable, performance, or the lab score. The statistical PLS analysis generated one significant component, displayed as the $y$ axis, describing $42 \%$ of the variance, $R^{2} y$, in lab score, with a predictive ability, $Q^{2}$, of $33 \%$. The statistics for this model are shown in Table II. Constructs that are close, i.e., on the same level horizontally, are positively correlated, while those that are on opposite sides of the $y$ axis are negatively correlated. Variables that are important in the model, and thus also important for predicting the lab report score, are located far from the origin of the figure. Thus, prior knowledge, sensemaking effort, problem solving confidence, and concentration emotions are positively correlated with each other and are important for the model's ability to predict students' 


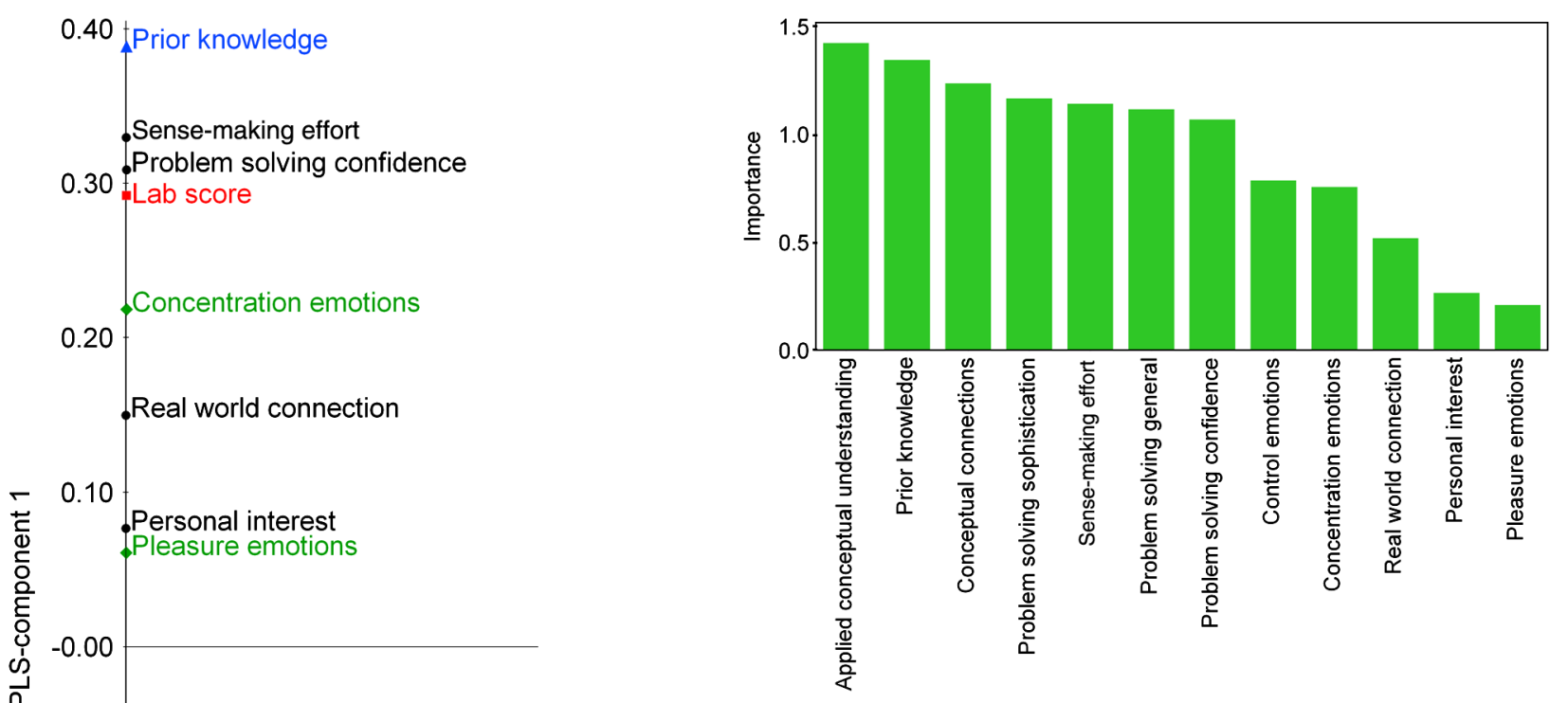

FIG. 2 (color online). Variables of importance in predicting (VIP) lab score. Values larger than 1 indicate important constructs while values lower than 0.5 indicate unimportant constructs.

performance, especially personal interest, which is surprising. Pleasure emotions are correlated with beliefs expressing personal interest, but show low correlation with how the students performed on the lab, implying that high quality performance is not driven by intrinsic motivation.

Figure 2 summarizes the relative importance of the measured constructs to model differences between students with respect to the independent variables as well as to predict the outcome variable lab score. The variables are presented in descending order; however, they do not in-

FIG. 1 (color online). PLS top model with one significant component ( $y$ axis) showing internal correlation between constructs as well as their relative importance for the models ability to predict the outcome variable lab score. Constructs that are close are positively correlated and those that are on the opposite side of the $y$ axis are negatively correlated. Constructs may contain positively as well as negatively polarized test items.

lab scores. The beliefs categories describing problem solving general, problem solving sophistication, conceptual connections, and applied conceptual understanding, which all express novicelike epistemological beliefs (see Table III in order to see how the items constitute each category), are also important predictors in the model and are all negatively correlated to lab score. The meaning of each base model is explained and analyzed in more detail below. This PLS top model also reveals that control emotions, which in this case are dominated by negative feelings of stress, ignorance, and frustration, are important for explaining performance and correlate negatively with lab score. Value beliefs, which are represented by real world connection and personal interest, show little correlation to dicate positive or negative correlation with performance. Values larger than 1 indicate important constructs while values lower than 0.5 indicate unimportant constructs.

\section{B. Description of constructs: Base models}

Items belonging to the same predefined construct were pooled into a base model and the internal dimensionality was investigated using PCA. The item's relative contribution to a student's score on a specific construct was weighted by their ability to measure the underlying variable (loading) in the base model. Table II presents a summary of the statistics of base models representing the different constructs that are used in Fig. 1.

Prior knowledge.-Students' responses to the 12 items in the conceptual physics test, together with the total score, were represented by a PCA base model expressed in one statistically significant component. The maximum score on this test was 12 and the average was 9.8. As shown in Fig. 2, prior knowledge in conceptual physics is the second most important variable for predicting performance.

Beliefs.-The responses from this 39-item questionnaire were categorized in the eight categories determined by 
TABLE III. Contributions (loadings) of beliefs items selected from the CLASS survey [32] to corresponding base models. Expert or novice indicates whether the base model represents expert or novicelike beliefs and correlates with lab score in Fig. 1.

Categories and items

Real world connection

35. To understand physics, I sometimes think about my personal experiences and relate them to the or novice topic being analyzed.

28. Reasoning skills used to understand physics can be helpful to me in my everyday life. 0.56

33. The subject of physics has little relation to what I experience in the real world. -0.54

$\begin{array}{ll}26 . & \text { Learning physics changes my ideas about how the world works. } \\ \end{array}$

Personal interest

26. Learning physics changes my ideas about how the world works.

Expert

13. I study physics to learn knowledge that will be useful in my life outside of school.

3. I think about the physics I experience in everyday life.

24. I enjoy solving physics problems.

28. Reasoning skills used to understand physics can be helpful to me in my everyday life.

10. I am not satisfied until I understand why something works the way it does.

Sense-making effort

30. Spending a lot of time understanding where formulas come from is a waste of time.

37. When I solve a physics problem, I explicitly think about which physics ideas apply to the problem.

34. There are times I solve a physics problem more than one way to help my understanding.

23. In physics, it is important for me to make sense out of formulas before I can use them correctly.

39. When studying physics, I relate the important information to what I already know rather than just memorizing it the way it is presented.

22. In doing a physics problem, if my calculation gives a result very different from what I'd expect,

I'd trust the calculation rather than going back through the problem.

10. I am not satisfied until I understand why something works the way it does.

0.58

0.52

0.45

0.31

0.26

0.11

$-0.43$

0.40

0.38

0.37

0.37

$-0.35$

Conceptual connections

30. Spending a lot of time understanding where formulas come from is a waste of time.

0.34

12. I do not expect physics equations to help my understanding of the ideas; they are just for doing calculations.

6. Knowledge in physics consists of many disconnected topics.

1. A significant problem in learning physics is being able to memorize all the information I need to know.

20. If I don't remember a particular equation needed to solve a problem on an exam, there's nothing much I can do (legally!) to come up with it.

5. After I study a topic in physics and feel that I understand it, I have difficulty solving problems on the same topic.

Applied conceptual understanding

38. If I get stuck on a physics problem, there is no chance I'll figure it out on my own.

1. A significant problem in learning physics is being able to memorize all the information I need to know.

20. If I don't remember a particular equation needed to solve a problem on an exam, there's nothing much I can do (legally!) to come up with it.

6. Knowledge in physics consists of many disconnected topics.

0.51

0.48

0.45

0.40

21. If I want to apply a method used for solving one physics problem to another problem, the problems must involve very similar situations.

7. When I solve a physics problem, I locate an equation that uses the variables given in the problem and plug in the values.

5. After I study a topic in physics and feel that I understand it, I have difficulty solving problems on the same topic.

Problem solving general

32. I can usually figure out a way to solve physics problems.

$-0.46$

0.47

0.46

0.41

0.40

0.35

0.35

$-0.06$
Novice

Expert

Expert

Novice

Novice

. 
TABLE III. (Continued)

\begin{tabular}{|c|c|c|}
\hline Categories and items & Loading & $\begin{array}{l}\text { Expert } \\
\text { or novice }\end{array}$ \\
\hline Real world connection & & Expert \\
\hline $\begin{array}{l}\text { 39. When studying physics, I relate the important information to what I already know rather than just } \\
\text { memorizing it the way it is presented. }\end{array}$ & -0.45 & \\
\hline 38. If I get stuck on a physics problem, there is no chance I'll figure it out on my own. & 0.42 & \\
\hline 24. I enjoy solving physics problems. & -0.42 & \\
\hline 15. Nearly everyone is capable of understanding physics if they work at it. & 0.30 & \\
\hline $\begin{array}{l}\text { 25. In physics, mathematical formulas express meaningful relationships among measurable } \\
\text { quantities. }\end{array}$ & -0.27 & \\
\hline $\begin{array}{l}\text { 12. I do not expect physics equations to help my understanding of the ideas; they are just for doing } \\
\text { calculations. }\end{array}$ & 0.25 & \\
\hline $\begin{array}{l}\text { 14. If I get stuck on a physics problem on my first try, I usually try to figure out a different way that } \\
\text { works. }\end{array}$ & 0.10 & \\
\hline Problem solving confidence & & Expert \\
\hline 32. I can usually figure out a way to solve physics problems. & 0.68 & \\
\hline 38. If I get stuck on a physics problem, there is no chance I'll figure it out on my own. & -0.62 & \\
\hline 15. Nearly everyone is capable of understanding physics if they work at it. & -0.38 & \\
\hline $\begin{array}{l}\text { 14. If I get stuck on a physics problem on my first try, I usually try to figure out a different way that } \\
\text { works. }\end{array}$ & 0.07 & \\
\hline Problem solving sophistication & & Novice \\
\hline $\begin{array}{l}\text { 20. If I don't remember a particular equation needed to solve a problem on an exam, there's nothing } \\
\text { much I can do (legally!) to come up with it. }\end{array}$ & 0.50 & \\
\hline 32. I can usually figure out a way to solve physics problems. & -0.49 & \\
\hline 38. If I get stuck on a physics problem, there is no chance I'll figure it out on my own. & 0.47 & \\
\hline 24. I enjoy solving physics problems. & -0.46 & \\
\hline $\begin{array}{l}\text { 5. After I study a topic in physics and feel that I understand it, I have difficulty solving problems on } \\
\text { the same topic. }\end{array}$ & 0.27 & \\
\hline $\begin{array}{l}\text { 21. If I want to apply a method used for solving one physics problem to another problem, the } \\
\text { problems must involve very similar situations. }\end{array}$ & 0.07 & \\
\hline
\end{tabular}

Adams et al. [32] and analyzed in separate PCA models. Not all test items in the questionnaire belonged to a category and some test items belonged to several categories as shown by Adams et al. The cross-validation indicated that the systematic variation among items in the different beliefs categories was difficult to predict, as shown by $Q^{2}$ in Table II. This could be due to the relatively small sample size. Given this, and the previous validation of the categories by Adams et al., we decided to calculate one component for each category, provided that the covariation structure among items within each category was consistent with theory. In Table III the individual test items belonging to each construct and their loadings are presented, showing their relative contribution to the first component of that particular base model as well as the polarization towards expertlike or novicelike representation for each base model.

Emotions.-The flow categories, control (control, calm, competent, ignorant, frustrated, stressed), concentration (concentration, focus, absorbed), and pleasure (interested, amused, motivated, elated), were used when creating the PCA base models representing emotions. The flow catego- ries thus gave three base models that all generated one significant component. In general, students expressed positive emotional experiences during the simulation. Averages of students' responses to the items in the pleasure and concentration categories were 3.6 and 3.8, respectively, on the five-degree Likert scale. The control category contained positive as well as negative items. Positive control emotions (control, calm, competent) have an average score of 3.3 while negative control emotions (ignorant, frustrated, and stressed) had an average of 3.6. For the control base model, the negative items were more important for describing the variance among students, thus defining the negative meaning of this construct (as expressed by the base model) and explaining the negative correlation with performance.

Lab score.-This base model, which represents performance, was generated from students' responses to the 15 questions in the lab instructions. As described earlier, students' responses were scored using the modified SOLO taxonomy. Analyzing scores with PCA produced one single significant lab score component. The subtasks showed an increasing complexity, from modeling of a 


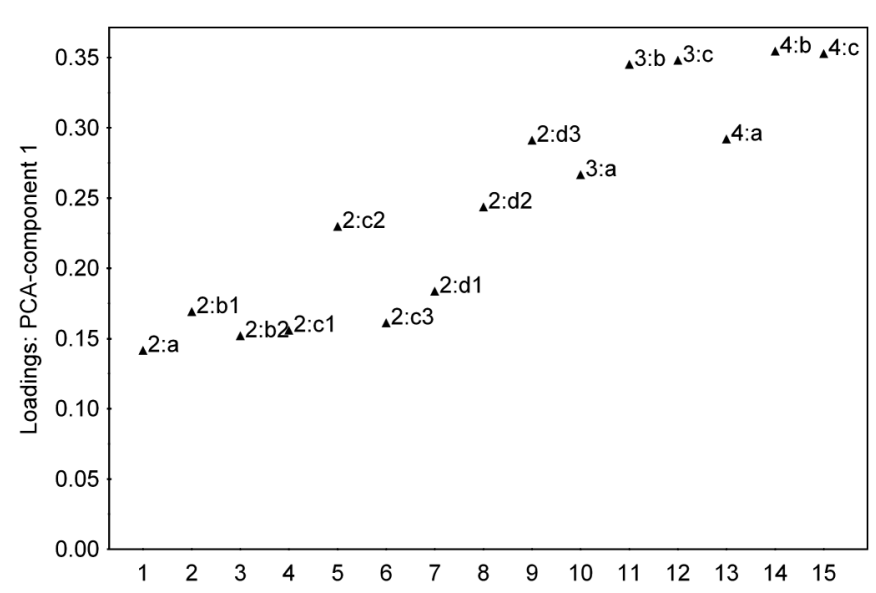

FIG. 3. PCA base model of lab score showing questions with increasing complexity, using one significant component ( $y$ axis). The $x$ axis indicates item index only and does not carry any further information about the data set.

small particle-spring system towards large particle-spring systems including damping. This increasing complexity, and hence discriminative ability, is reflected in this base model (Fig. 3) where responses to questions in the third and fourth subtasks generate larger variance among students, and thus have the highest loadings in the model.

\section{DISCUSSION}

Numerical problem solving in physics education is, compared to traditional problem solving, a complex learning situation where knowledge and skills in several disciplines are integrated. Furthermore, as in the present case, the degree of freedom is often high, allowing students to plan and conduct their work in accordance with, for example, their prior knowledge, own values, beliefs about learning, and priorities pertinent to their learning needs and goals. As mentioned earlier, motivation to engage in an activity, and thus the quality of outcomes, depends on how well situational factors align with the individual's goals, skills, and values. Thus, it is not self-evident that a situation with a high degree of freedom will appeal to and offer good learning opportunities for all types of students. To be able to predict the efficiency of specific learning activities to motivation and cognition, we need a better understanding of the complex array of variables that are involved in the appraisal processes and their interactions with motivation and cognition. In the present study, several of the measured characteristics play important roles in predicting the students' performance of the task. Our results show that the students' prior knowledge and epistemological beliefs, together with control and concentration emotions, are important variables for predicting performance, while evaluations of the utility of physics knowledge in everyday life, i.e., value beliefs, or the extent to which students enjoyed working with the simulation, pleasure emotions, are not correlated with performance as much.

\section{A. Prior knowledge}

Although the task did not actually introduce any new physics, in addition to the basic-level classical mechanics that formed the context of the problem and should be familiar to the students, prior knowledge in physics was among the most important predictors of performance. This is not so surprising since there is a well-established link between structure of prior knowledge and performance on complex tasks. Students who have well-developed schemata in the subject area not only have better opportunities to quickly overview the task and identify key issues and possible strategies to solve the task, but will also experience a reduced load on working memory, due to information chunking, which allows for more creative thinking. This would certainly be the case in any learning situation, but we argue that the need for relevant and well-structured prior knowledge is accentuated in situations with a high degree of freedom like in the present study. This finding supports the idea that students with good prior knowledge to a relatively high extent express feelings of control and calmness while less knowledgeable students experience higher levels of frustration and stress, as well as feeling less competent. Furthermore, the importance of prior physics knowledge for successful performance in this study indicates that the task did require significant interaction with conceptual physics and thus had potential effects on the development of students' physics understanding, although the task was considered by the teachers mainly as a programming exercise.

\section{B. Beliefs about physics and learning}

Because of the short time scales in this study we assume that students' beliefs did not change during the time of the study. Even though previous research has shown that shortterm intervention using a refutational instruction approach can possibly change students' epistemologies [45] towards a more sophisticated view, it is not clear how stable these changes are. Other studies have shown that possible changes in students' epistemologies usually need longer periods of time, e.g., semester or even years $[9,46]$. Our results showed that students' initial beliefs about physics did predict performance. In fact, novicelike beliefs that physics knowledge consists of isolated pieces of information that are to be remembered and cannot be developed further by the student (i.e., beliefs found in applied conceptual understanding) were even more important than prior knowledge to explain the quality of the lab report. This belief was associated with low performance on the lab report, but also a low perceived degree of control over the situation, low perceived competence, and low scores on the conceptual pretest. Hence, beliefs indicating a tendency to solve physics problems without elaboration of links between formulas and theory, i.e., the novicelike beliefs found in problem solving sophistication and problem solving general, have predicted performance as well as 
affective experiences negatively in this learning situation, while an inclination to connect what is learned with prior knowledge and to understand underlying principles rather than to mechanically apply formulas (as indicated by sense-making effort) was positively associated with performance, control, and sense of competence.

In this study, we have measured performance, lab score, in terms of complexity of student's reasoning in their answers to questions in the lab manual. As shown in Fig. 3, questions that offer, but not explicitly require, possibilities for more complex answers are those that are most important for describing differences in student performance, and thus more likely to predict performance. Since the required level of complexity was not specified in these questions, we argue that this measure reflects students' choice of level for complex reasoning. Our results indicate that the choice to engage in a higher-level reasoning is not only related to the students' ability for high complexity reasoning, i.e., having appropriate knowledge in the subject area, but also to the students' beliefs about how physics should be learned and, implicitly, the nature of physics knowledge, at least when the situation provides scope for such decisions. However, the positive correlation between students' expertlike beliefs and a high level of prior knowledge, as shown in Fig. 1, could be interpreted as students' beliefs about physics and learning have had impact on their learning in general, i.e., the character of the physics knowledge they have developed prior to the course. Thus, there is a possibility that the predictive ability of students' beliefs on performance is mediated by the level of prior knowledge. Novicelike beliefs were associated with feelings of frustration, stress, and low perceived competence, while students with expertlike beliefs to a larger extent than others instead experienced a sense of being in control of the situation and calmness. This is in line with the assumption that students who believe that learning physics is about understanding the large picture, e.g., principles, and interrelations, rather than memorizing isolated facts, would have developed more coherent and complex prior schemata in the subject area, thus reducing risk for cognitive overload by facilitating overview and processing of the complex physics task [47].

\section{Emotional experiences}

As mentioned earlier, state emotions have been hypothesized to play several different roles in motivational processes [35]. Emotions can serve as indicators of how well the situation matches the individual's beliefs, values, skills, as well as measures of progress and perceived control, and thus provide valuable information of the efficiency of a learning situation. In the present study, three different types of emotions were measured, pleasure, control, and concentration, in order to investigate their relationship with performance and students' beliefs about physics. These types of emotions have been used within research on flow but also have bearing on, for example, the intrinsic-extrinsic continuum in self-determination theory. Our results show that students' emotional experiences during the exercise are subordinate to students' epistemological beliefs and prior knowledge for predicting performance. Only concentration and control emotions had some correlation with performance, while pleasure emotions were unimportant for predicting performance.

Internalization and identification of external goals, norms, and values is considered to result in extrinsic motivation with a more intrinsic character, whose development is proposed to be associated with autonomy and feelings of competence and control. In this case, we propose that feelings of competence were achieved in those cases when situational demands matched students' personal characteristics, of which epistemological beliefs and physics knowledge had most influence. We suggest that students' epistemological beliefs did function as goals (cf. Carver and Scheier's reference values [11]) that provided direction for their behavior. According to selfdetermination theory, internalization of external goals, i.e., expertlike beliefs, would not necessarily lead to enjoyment emotions. However, when goals are integrated, behavior is to a high extent perceived as self-regulated and manifested in a sense of control and competence if goals are adaptive in the specific situation.

One conclusion that could be drawn from this is that learning not necessarily has to be driven by intrinsic motivation, i.e., to be fun. However, since affective memories are considered as one of the major contributors to attitudes, and thus future choice behavior, it would be a mistake not to consider the intrinsically motivating properties of learning situations if we want the students to enroll in future physics courses.

\section{CONCLUSIONS}

The students' prior knowledge and epistemological beliefs, together with control and concentration emotions, have been shown to be important variables for predicting performance, while evaluations of the utility of physics knowledge in everyday life, i.e., value beliefs, or the extent to which students enjoyed working with the simulation, pleasure emotions, do not show the same predictive ability on performance in the particular context of this study.

An environment for numerical problem solving can be used for solving challenging and realistic physics problems, providing a learning situation with high degree of freedom. The particle-spring model system used in this study can be used to model and simulate many realistic and interesting phenomena and thus function as a context for motivated learning behavior. However, our results indicate that although a certain degree of enjoyment is probably necessary, integration and identification of expertlike beliefs about learning are more strongly associated with concentration and control during learning and, ultimately, 
with high performance. In this situation with many degrees of freedom, participants who believed that difficult problems can be solved by investing more effort and acknowledged their own responsibility in learning performed better and experienced less frustration and stress than those who were more authority dependent and showed less confidence in their ability to solve problems. This has two consequences: (1) in order to improve student's performance, and hence confidence in problem solving, the situational demands, e.g., in terms of degrees of freedom, should be adjusted to meet the beliefs of the students, and (2) in order to help our students to develop their ability to cope with situations that require them to take charge of their own learning, it is necessary to also focus on the development of expertlike beliefs about learning as well as their content knowledge. We argue that this development could be stimulated by using tasks with high degree of freedom or challenging tasks, such as the numerical physics problem used in this study, where expertlike beliefs are required for successful performance.

It should be pointed out that the study was performed in one specific setting. Thus, although the data clearly support the conclusions made, there is a need for further research to test the relationship between students' beliefs, emotional experiences, and performance in other settings before wider generalizations can be made. The motivational and cognitive processes that influences learning are multifaceted and complex. Hence, to gain a better understanding of how different learning situations can support learning for different types of students, studies that simultaneously consider relationships between many relevant variables and their relative importance for learning are strongly warranted.

\section{ACKNOWLEDGMENTS}

This work is supported by the Swedish National Graduate School in Science and Technology Education.

\section{APPENDIX: CONTRIBUTIONS OF BELIEF ITEMS}

In Table III the individual test items from the CLASS questionnaire by Adams et al. [32] belonging to each construct and their loadings are presented, showing their relative contribution to the first component of that particular base model as well as the polarization towards expertlike or novicelike representation for each base model.
[1] Marty Johnston, Implementing curricular change, Comput. Sci. Eng. 8, 32 (2006).

[2] Rubin Landau, Computational physics: A better model for physics education?, Comput. Sci. Eng. 8, 22 (2006).

[3] Ruth Chabay and Bruce Sherwood, Computational physics in the introductory calculus-based course, Am. J. Phys. 76, 307 (2008).

[4] Wolfgang Christian and Francisco Esquembre, Modeling physics with easy JAVA simulations, Phys. Teach. 45, 475 (2007).

[5] Edward F. Redish and Jack M. Wilson, Student programming in the introductory physics course: M.U.P.P.E.T., Am. J. Phys. 61, 222 (1993).

[6] Ross L. Spencer, Teaching computational physics as a laboratory sequence, Am. J. Phys. 73, 151 (2005).

[7] David Scherer, Paul Dubois, and Bruce Sherwood, VPYTHON: 3D interactive scientific graphics for students, Comput. Sci. Eng. 2, 56 (2000).

[8] R. M. Ryan and E. L. Deci, Intrinsic and extrinsic motivations: Classic definitions and new directions, Contemp. Educ. Psychol. 25, 54 (2000).

[9] B. K. Hofer and P. R. Pintrich, The development of epistemological theories: Beliefs about knowledge and knowing and their relation to learning, Rev. Educ. Res. 67, 88 (1997).

[10] Mark Windschitl and Thomas Andre, Using computer simulations to enhance conceptual change: The roles of constructivist instruction and student epistemological beliefs, J. Res. Sci. Teach. 35, 145 (1998).
[11] C. S. Carver and M.F. Scheier, Origins and functions of positive and negative affect: A control-process view, Psychol. Rev. 97, 19 (1990).

[12] P. A. Schutz and J.T. DeCuir, Inquiry on emotions in education, Educ. Psychol. 37, 125 (2002).

[13] J. Osborne, S. Simon, and S. Collins, Attitudes towards science: A review of the literature and its implications, Int. J. Sci. Educ. 25, 1049 (2003).

[14] Allan Wigfield and Jacquelynne S. Eccles, Expectancy value theory of achievement motivation, Contemp. Educ. Psychol. 25, 68 (2000).

[15] David Hestenes, Toward a modeling theory of physics instruction, Am. J. Phys. 55, 440 (1987).

[16] Madelen Bodin, Kenneth Bodin, and Emil Ernerfelt, "Creative simulation environments for physics education" (unpublished).

[17] N. Chonacky and D. Winch, 3Ms for instruction, Part 2: MAPLE, MATHEMATICA, and MATLAB, Comput. Sci. Eng. 7, 14 (2005).

[18] Phil Benson, Teaching and Researching Autonomy in Language Learning, Applied Linguistics in Action Series (Pearson Education Limited, London, 2001).

[19] Johnmarshall Reeve, Elizabeth Bolt, and Yi Cai, Autonomy-supportive teachers: How they teach and motivate students, J. Educ. Psychol. 91, 537 (1999).

[20] Teresa K. DeBacker, H. Michael Crowson, Andrea D. Beesley, Stephen J. Thoma, and Nita L. Hestevold, The challenge of measuring epistemic beliefs: An analysis of 
three self-report instruments, J. Exp. Educ. 76, 281 (2008).

[21] Barbara K. Hofer and Paul R. Pintrich, Personal Epistemology: The Psychology of Beliefs about Knowledge and Knowing (Erlbaum Associates, Mahwah, NJ, 2002).

[22] William G. Perry, Forms of Intellectual and Ethical Development in the College Years: A Scheme (Holt, Rinehart and Winston, New York, 1970).

[23] M. Schommer, Effects of beliefs about the nature of knowledge on comprehension, J. Educ. Psychol. 82, 498 (1990).

[24] David Hammer, Epistemological beliefs in introductory physics, Cogn. Instr. 12, 151 (1994).

[25] T. Mikael Winberg and C. Anders R. Berg, Students' cognitive focus during a chemistry laboratory exercise: Effects of a computer-simulated prelab, J. Res. Sci. Teach. 44, 1108 (2007).

[26] Andrew Elby and David Hammer, On the substance of a sophisticated epistemology, Sci. Educ. 85, 554 (2001).

[27] Edward F. Redish, Millikan lecture 1998: Building a science of teaching physics, Am. J. Phys. 67, 562 (1999).

[28] D. C. Finster, Developmental instruction: Part 1. Perry's model of intellectual development, J. Chem. Educ. 66, 659 (1989).

[29] David C. Finster, Developmental instruction: Part II. Application of the Perry model to general chemistry, J. Chem. Educ. 68, 752 (1991).

[30] Cynthia R. Hynd, Mary M. McNish, Gaoyin Qian, Mark Keith, and Kim Lay, Reading Research Report No. 16 (National Reading Research Center, Athens, GA, 1994).

[31] Kit-Ling Lau and John Lee, Examining Hong Kong students' achievement goals and their relations with students' perceived classroom environment and strategy use, Educ. Psychol. Rev. 28, 357 (2008).

[32] W. K. Adams, K. K. Perkins, N. S. Podolefsky, M. Dubson, N. D. Finkelstein, and C. E. Wieman, New instrument for measuring student beliefs about physics and learning physics: The Colorado Learning Attitudes about Science Survey, Phys. Rev. ST Phys. Educ. Res. 2, 010101 (2006).

[33] Dale H. Schunk, Paul R. Pintrich, and Judith L. Meece, Motivation in Education: Theory, Research, and Applications (Pearson Education, Upper Saddle River, NJ, 2008), 3rd ed.
[34] M. Csikszentmihalyi and J. LeFevre, Optimal experience in work and leisure, J. Pers. Soc. Psychol. 56, 815 (1989).

[35] Reinhard Pekrun, Thomas Goetz, Wolfram Titz, and Raymond P. Perry, Academic emotions in students' self-regulated learning and achievement: A program of qualitative and quantitative research, Educ. Psychol. 37, 91 (2002).

[36] R. M. Ryan, J. Stiller, and J. H. Lynch, Representations of relationships to teachers, parents, and friends as predictors of academic motivation and self-esteem, J. Early Adolesc. 14, 226 (1994).

[37] Mihály Csíkszentmihályi, Flow: The Psychology of Optimal Experience (Harper \& Row, New York, 1990), 1 st ed.

[38] D. Hestenes, M. Wells, and G. Swackhamer, Force Concept Inventory, Phys. Teach. 30, 141 (1992).

[39] Ronald K. Thornton and David R. Sokoloff, Assessing student learning of Newton's laws: The force and motion conceptual evaluation and the evaluation of active learning laboratory and lecture curricula, Am. J. Phys. 66, 338 (1998).

[40] J. A. Ghani and S. P. Deshpande, Task characteristics and the experience of optimal flow in human-computer interaction, J. Psychol. 128, 381 (1994).

[41] John B. Biggs and Kevin F. Collis, Evaluating the Quality of Learning: The SOLO Taxonomy (Structure of the Observed Learning Outcome), Educational Psychology (Academic, New York, 1982).

[42] I. T. Joliffe, Principal Component Analysis (SpringerVerlag, New York, 1986).

[43] Umetrics, SIMCA-P+, 2009.

[44] H. Eastment and W. Krzanowski, Cross-validatory choice of the number of components from a principal component analysis, Technometrics 24, 73 (1982).

[45] Dorothe Kienhues, Rainer Bromme, and Elmar Stahl, Changing epistemological beliefs: The unexpected impact of a short-term intervention, Br. J. Educ. Psychol. 78, 545 (2008).

[46] C. A. Ogilvie, Changes in students' problem-solving strategies in a course that includes context-rich, multifaceted problems, Phys. Rev. ST Phys. Educ. Res. 5, 020102 (2009).

[47] J. Sweller, J. van Merrienboer, and F. Paas, Cognitive architecture and instructional design, Educ. Psychol. Rev. 10, 251 (1998). 